\title{
A New Residual Life Prediction Method for Complex Systems Based on Wiener Process and Evidential Reasoning
}

\author{
Xin Zhang, ${ }^{1}$ Shuaiwen Tang, ${ }^{1}$ Taoyuan Liu, ${ }^{1}$ and Bangcheng Zhang $\mathbb{D}^{2}$ \\ ${ }^{1}$ Rocket Force University of Engineering, Xi'an 710000, China \\ ${ }^{2}$ School of Mechatronic Engineering, Changchun University of Technology, Changchun 130012, China \\ Correspondence should be addressed to Bangcheng Zhang; zhangbangcheng@ccut.edu.cn
}

Received 21 February 2018; Accepted 26 March 2018; Published 3 May 2018

Academic Editor: Youqing Wang

Copyright (c) 2018 Xin Zhang et al. This is an open access article distributed under the Creative Commons Attribution License, which permits unrestricted use, distribution, and reproduction in any medium, provided the original work is properly cited.

\begin{abstract}
A new residual life prediction method for complex systems based on Wiener process and evidential reasoning is proposed to predict the residual life of complex systems effectively. Moreover, the better maintenance strategies and decision supports are provided. For the residual life prediction of complex systems, the maximum likelihood method is adopted to estimate the drift coefficient, and the Bayesian method is adopted to update the parameters of Wiener process. The process of parameters estimation and the probability density function (PDF) of the residual life are deduced. To improve the accuracy of the residual life prediction results, the evidential reasoning (ER) is used to integrate the prediction results of Wiener process. Finally, a case study of gyroscope is examined to illustrate the feasibility and effectiveness of the proposed method, compared with fuzzy theory, which provides an important reference for the optimization of the reliability of complex systems and improvement.
\end{abstract}

\section{Introduction}

Nowadays, the residual life prediction is becoming an attention and hot topic in engineering practice. Especially in the performance study of pipeline, oil depot, engine, LED lamp control system, storage tanks, gyroscope, and other complex systems, the residual life prediction has been widely applied. If the residual life of complex systems can be estimated at the beginning of the performance degradation of the systems, and if the performance can be evaluated effectively [1], especially when no major damage has been caused, the happening of unnecessary faults will be avoided to some extent and the maintenance costs will be reduced greatly [2]. Therefore, it is essential to study the residual life prediction of complex systems.

In the traditional life experiment, failure data can be used to predict the life of equipment and device. On the one hand, many failure data cannot be obtained in a short time [3]. On the other hand, some of the systems' characteristics will degrade over time. However, a large amount of information related to reliability and residual life is included in the degradation data. Therefore, the degradation data can be used to predict the residual life of complex systems. Through the acceleration test, both reliability and life information under different environmental conditions can be obtained. At present, the commonly used acceleration equations include Arrhenius model, Eyring model, Power Law Inverse (IPL), and Hallberg-Peck model. Among them, Arrhenius and Eyring models are always used for thermal accelerated stress in the systems, while temperature is the major factor causing aging. IPL is always used for other different kinds of accelerated stresses except thermal accelerated stress. HallbergPeck model synthesizes temperature and humidity that can describe the aging test more accurately under conditions of temperature and humidity.

In current study, the prediction methods can be classified as the physics-based prediction method [4] and data-driven prediction method [5]. With the development of related technologies such as signal acquisition and signal processing, abundant data of system operation can be obtained. According to these data, the corresponding mathematical model is utilized. The method is mainly composed of two kinds of techniques, which are named as artificial intelligence and probability statistics. The artificial intelligence methods have 


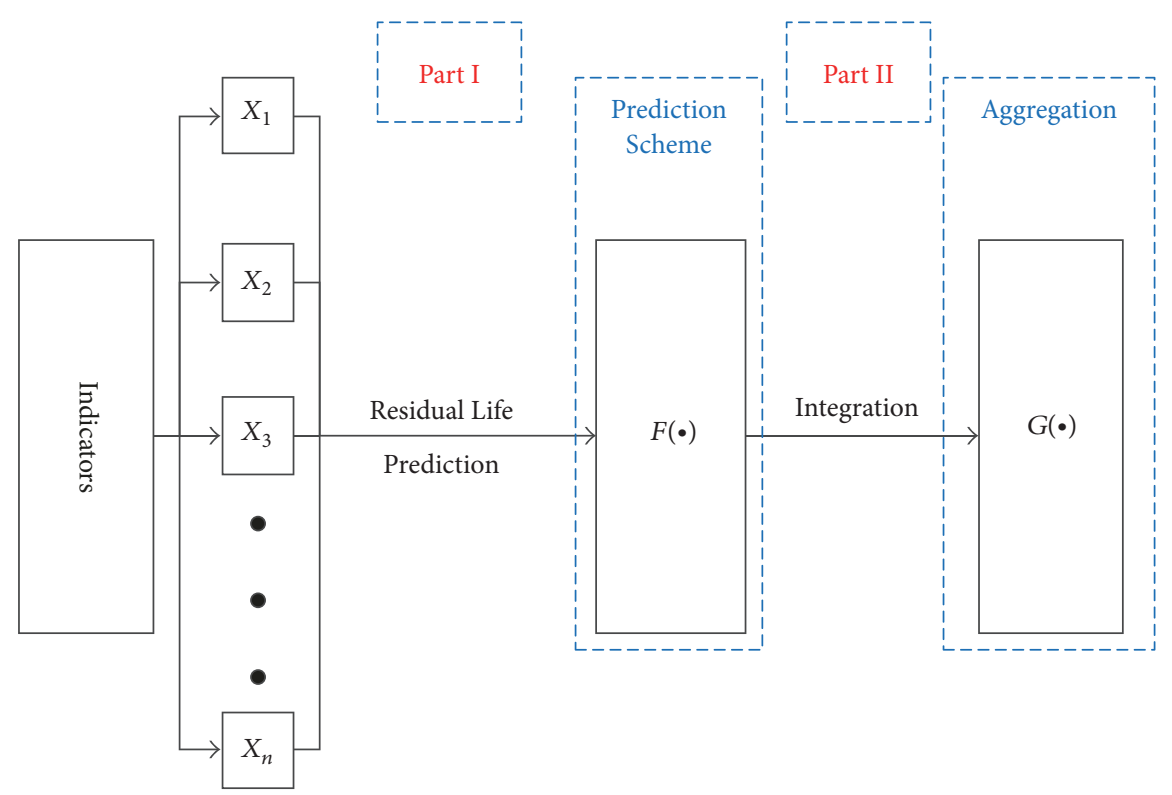

FIGURE 1: Main structure of the new residual life prediction model.

a higher degree of the data fitting, but they cannot obtain the uncertainty of the prediction. The probability statistics can predict the future state better.

Firstly, Wiener process is a kind of the most commonly used probability statistical model, which can describe the degradation process accurately $[6,7]$. The degradation model based on Wiener process has obvious advantages in mathematics [8]. Wiener process has been widely applied in many fields [9-11]. Wang proposed a reliability assessment method that fused both prior and on-site degradation data [12]. To update the parameters of Wiener process, the prior data was used to determine the parameters, and the on-site data was integrated by Bayesian method. In [13], the degradation of aeroengine was modeled based on multistage Wiener process. Based on the historical degradation data and the historical failure time data, the prior distribution of the model parameters was estimated by using the expectation maximization (EM) algorithm. In [14], the recursive filtering algorithm and EM algorithm were combined to update the parameters of the Wiener process, and the method was applied to the inertial navigation system to predict the residual life accurately.

Secondly, the evidential reasoning rule (ER rule) proposed by Yang and Xu [15] in 2013 is also a kind of probabilistic statistical model. As a multiattribute decision-making method, the ER rule is widely used to solve the fuzziness and uncertainty of parameters in the evaluation and decisionmaking process. In $[16,17]$, the authors have described how to handle the multiattribute decision analysis under uncertainty by the ER rule that is developed based on the decision theory and D-S theory of evidence [15]. Meanwhile, the ER rule is suitable for solving and expressing uncertainty [18-20].

To solve above problems, the prediction framework of the residual life of complex systems is constructed, and the corresponding model of complex systems based on Wiener process and evidential reasoning is established in this paper. The other contents of this paper are mainly organized as follows. In Section 2, the structure of the residual life prediction model of complex systems is constructed, and the problems to be solved are described in detail. The residual life prediction model of complex systems based on Wiener process and evidential reasoning is established in Section 3, and the details of modeling steps are organized and summarized systematically. In the Section 4, a case study about the residual life prediction of gyroscope is done, based on fuzzy theory and evidential reasoning, respectively, and the simulation results are analyzed. Finally, the practicability of the prediction methods proposed and future work are summarized in the Section 5, as well as the reference of other similar products.

\section{New Residual Life Prediction Model and Problem Formulation}

2.1. Structure of the New Residual Life Prediction Model. The residual life of complex systems is influenced by many factors. They can be divided into two categories, which are named as the internal factors and the external factors, respectively. The internal factors are composed of materials, structure, and so on, while the external factors include debugging, operation, and maintenance. Thus, it is hard and complex to establish an accurate and complete mathematical prediction model of residual life of complex systems [21]. However, the residual life of the systems can be reflected by some so-called health indicators [22]. Suppose $x_{i}$ denotes the $i$ th health indicator for the monitoring, $i=1,2, \ldots, n$. By integrating all the health indicators, a new prediction model of residual life is established, whose structure is shown as Figure 1.

Figure 1 shows the structure of the new model that mainly includes two parts. In the first part, the residual life prediction 
model is established based on the selected health indicators and Wiener process. In the second part, the prediction results are integrated by the ER rule to obtain more accurate prediction results.

2.2. Problem Description for Residual Life Prediction. Based on Figure 1, the following three problems should be studied in order to predict the residual life of complex systems.

Problem 1. In Figure 1, the prediction of residual life is necessary. Thus, it is essential to establish a prediction model to get the indicators' prediction value with the obtained indicators' information. Therefore, Problem 1 concentrates on how to establish the following model:

$$
\bar{y}_{i}(t)=F\left(x_{i}(1), x_{i}(2), \ldots, x_{i}(n)\right),
$$

where $i=1,2, \ldots, n \cdot \bar{y}_{i}(t)$ denotes the prediction value of the residual life of indicator $x_{i}$, which is a time series in nature. $F(\bullet)$ represents a nonlinear function.

Problem 2. In practice, in order to represent the relative importance of the health indicators, it is of much necessity to allocate an adaptive weight to each indicator. Thus, Problem 2 concentrates on how to establish the following model to adjust the weight and update the weight coefficient according to the whole available observation data of all indicators:

$$
\omega_{i}(k)=\Psi\left(x_{i}(1), x_{i}(2), \ldots, x_{i}(n)\right),
$$

where $\sum_{i=1}^{n} \omega_{i}(k)=1 . \Psi(\bullet)$ represents a nonlinear function to determine the weighting coefficient.

Problem 3. To obtain the comprehensive prediction results, it is necessary to integrate various indicators and reprocess the prediction value of the residual life. Therefore, Problem 3 concentrates on how to establish the following integration model to obtain the residual life level of complex systems:

$$
g(i)=G\left(\omega_{i}(1), \omega_{i}(2), \ldots, \omega_{i}(n), \bar{y}_{i}(t)\right),
$$

where $G(\bullet)$ represents a nonlinear function.

Based on the above three problems, the concrete models are shown as follows.

\section{Residual Life Prediction Model}

In this paper, the following two models are used to solve the above three problems. Firstly, based on Wiener process, a degradation model of complex systems is established to analyze and describe the performance degradation process. In order to eliminate the tendency items, the incremental degradation is adopted. Secondly, the parameters of the degradation model are estimated and updated, with the maximum likelihood method to estimate the parameters, and the Bayesian method to update the parameters. Thirdly, the residual life prediction model of complex systems is established based on evidential reasoning, compared with fuzzy theory, and the prediction results of Wiener process are integrated to obtain more accurate prediction results.

\subsection{Prediction Model of Residual Life of Complex Systems Based on Wiener Process}

3.1.1. Degradation Model of Complex Systems. Wiener process has been widely used in the modeling of degradation process because of the excellent capability of analyzing and describing the process of degradation $[23,24]$. So, it is used to model the performance of complex systems in this paper.

If $Z(t)$ is the degradation measure of the performance of the systems at time $t$, the degradation model of Wiener process based on the drift parameter is

$$
\Delta Z(t)=Z(t+\Delta t)-Z(t)=\theta \Delta t+\varepsilon \Delta W(t),
$$

where $\theta$ is drift coefficient, $\varepsilon$ is diffusion coefficient, $\Delta t$ is a short time interval, and $W(t)$ is the Standard Brownian Motion which has two features as follows.

Feature 1. $W(t) \sim N(0, t)$.

Feature 2. For any two different times $t_{1}$ and $t_{2}, t_{1}<t_{2}, t_{1}+$ $\Delta t<t_{2}, \Delta W\left(t_{1}\right)$, and $\Delta W\left(t_{2}\right)$ are independent.

According to the characteristic of $W(t)$, it is assumed that $\Delta Z(t)$ obeys the normal distribution, that is, $\Delta Z(t) \sim$ $N\left(\theta \Delta t, \varepsilon^{2} \Delta t\right)$. So, its degradation amount mean is $E(\Delta Z(t))=$ $\theta \Delta t$, and degradation amount variance is $D(\Delta Z(t))=$ $\varepsilon^{2} \Delta t$. The mean reflects the trend of the degradation data affected by the accelerated stress. So, it is considered that the drift coefficient $\theta$ is related to the acceleration stress. The variance reflects the degree of deviation between the degenerate data and the mean, which is usually unrelated to the acceleration stress. So it is considered that the diffusion coefficient $\varepsilon$ has nothing to do with the acceleration stress. In this paper, the random parameters $\theta$ and $\varepsilon^{2}$ are used to describe the individual difference of complex systems. Since there is no sufficient prior information of the parameters to be estimated, the prior distribution without information is adopted.

The failure threshold of the performance of some complex systems is often a fixed value, which is set as a constant $C$. When the current degradation data reaches the threshold value for the first time, the system is failed. So, its residual useful life can be defined as

$$
\gamma=\inf \{t \mid \Delta Z(t) \geq C, t>0\} .
$$

It is proved that its first failure threshold time $\gamma$ is subject to inverse Gaussian distribution, and its probability density function is

$$
f(t \mid \theta, \varepsilon)=\frac{C-\Delta Z(t)}{\sqrt{2 \pi \varepsilon^{2} t^{3}}} \exp \left[-\frac{(C-\Delta Z(t)-\theta t)^{2}}{2 \varepsilon^{2} t}\right] .
$$

And the cumulative distribution function is

$$
\begin{aligned}
F(t)= & \phi\left(\frac{\theta t-\Delta Z(t)-C}{\varepsilon \sqrt{t}}\right) \\
& +\exp \left(\frac{2 \theta C}{\varepsilon^{2}}\right) \phi\left[\frac{-(\theta t-\Delta Z(t))+C}{\varepsilon \sqrt{t}}\right],
\end{aligned}
$$


where $\phi(\bullet)$ is probability density function of the standard normal distribution.

In the accelerated degradation test, temperature and humidity are the most common accelerated stresses that can aggravate the reaction to degenerate the product. Both temperature and humidity are important factors for reliability of some complex systems. High temperature and humid environment may make the service life decrease, so the entire life of the system is seriously affected. In this way, the Hallberg-Peck acceleration model is used to construct the relationship between the drift coefficient with the temperature and humidity stresses. Considering the influence of temperature and humidity comprehensively, the HallbergPeck model can describe the accelerated degradation test of the product under the condition of temperature and humidity accurately. Its expression is

$$
\theta=A\left(H_{R}\right)^{-3} \exp \left[\frac{E}{K(273+T)}\right],
$$

where $A$ is a constant, $A>0, H_{R}$ is relative humidity, and $E$ is activation energy in the unit of electron volts $(\mathrm{eV}) . K$ is Boltzmann constant whose value is $8.617 \times 10^{-5} \mathrm{eV} /{ }^{\circ} \mathrm{C}$. So, the unit of $E / K$ is ${ }^{\circ} \mathrm{C}$. In addition, $T$ is relative temperature. The degradation model can be obtained through substituting (8) into (4) as

$$
\begin{aligned}
\Delta Z\left(t, T, H_{R}\right)= & A\left(H_{R}\right)^{-3} \exp \left[\frac{E}{K \cdot(273+T)}\right] \Delta t \\
& +\varepsilon \Delta W(t)
\end{aligned}
$$

3.1.2. The Estimation of Parameters. The accelerated stresses in the accelerated degradation test are set to $T_{1}, T_{2}, \ldots, T_{l}$. There are six samples for each acceleration stress, and the measured time gradient of each sample is $t_{1}, t_{2}, \ldots, t_{n}$. The degradation amount of the $j$ th sample under the $T_{i}$ stress is $Z_{i j k}$ at time $t_{k}$, and the performance degradation is 0 at the initial time; $\Delta Z_{i j k}=Z_{i j k}-Z_{i j(k-1)}$ is the increment of degradation. By the nature of $W(t), \Delta Z_{i j k}(t) \sim N\left(\theta_{i j} \Delta t_{i j k}, \varepsilon^{2} \Delta t_{i j k}\right)$, we have

$$
\begin{aligned}
E\left(\Delta Z_{i j k}\right) & =\theta_{i j} \Delta t_{i j k} \\
& =A\left(H_{R}\right)^{-3} \exp \left[\frac{E}{K \cdot(273+T)}\right] \Delta t_{i j k} \\
D\left(\Delta Z_{i j k}\right) & =\varepsilon^{2} \Delta t_{i j k} .
\end{aligned}
$$

In the period of $\Delta t_{k}$, the probability density function (PDF) of the performance degradation data is

$$
D\left(\Delta Z_{i j k}\right)=\varepsilon^{2} \Delta t_{i j k}
$$

The likelihood function of $\theta$ and $\varepsilon^{2}$ can be obtained in

$$
\begin{aligned}
& L(A, E, \varepsilon) \\
& \quad=\prod_{i=1}^{l} \prod_{j=1}^{m} \prod_{k=1}^{n} \frac{1}{\sqrt{2 \pi \varepsilon^{2} \Delta t_{i j k}}} \exp \left[-\frac{\left(\Delta Z_{i j k}-\theta \Delta t_{i j k}\right)^{2}}{2 \varepsilon^{2} \Delta t_{i j k}}\right] .
\end{aligned}
$$

The maximum likelihood estimation can be used to estimate the values of $\theta$ and $\varepsilon^{2}$ in (13). But the solving process is very complicated. Therefore, the prior distribution of the parameters is determined firstly, and the parameters are updated by the Bayesian method secondly; then the distribution of the parameters can be obtained. Based on the Bayesian theory, the posterior distribution can be represented as

$$
p(\theta \mid y) \propto f(y \mid \theta) p(\theta)
$$

where $p(\theta \mid y)$ is the probability density function of posterior distribution, $f(y \mid \theta)$ is likelihood function, and $p(\theta)$ is the probability density function of prior distribution.

If the distribution of the measured data of the first set sample in stress $T_{1}$ is a normal distribution, where the parameters of $\theta_{1}$ and $\varepsilon_{1}^{2}$ are noninformative prior distribution, the prior distribution probability density function can be expressed as

$$
p\left(\theta, \varepsilon^{2}\right) \propto \frac{1}{\varepsilon^{2}}
$$

Let $\theta_{a}=\theta_{1} \Delta t_{11 k}, \varepsilon_{a}^{2}=\varepsilon_{1}^{2} \Delta t_{11 k}$, then $\varepsilon_{a}^{2}=\varepsilon_{1}^{2} \Delta t_{11 k}$, the joint posterior distribution of $\left(\theta_{a}, \varepsilon_{a}^{2}\right)$ is:

$$
\begin{aligned}
& p\left(\theta_{a}, \varepsilon_{a}^{2} \mid \Delta Z_{11 k}\right) \\
& \propto\left(\varepsilon_{a}^{2}\right)^{-n / 2-1} \exp \left[\sum_{k=1}^{n}-\frac{1}{2 \varepsilon_{a}^{2}}\left(\Delta Z_{11 k}-\theta_{a}\right)^{2}\right]
\end{aligned}
$$

First of all, to update the parameters of the estimated parameters $\theta_{a}, \varepsilon_{a}^{2}$ can be regarded as redundant parameters. To integrate the posterior distribution of the integral, the excess parameters are removed, shown in

$$
\begin{aligned}
& p\left(\theta \mid \Delta Z_{11 k}\right) \\
& \propto \int_{0}^{\infty}\left(\varepsilon_{a}^{2}\right)^{-n / 2-1} \exp \left[\sum_{k=1}^{n}-\frac{1}{2 \varepsilon_{a}^{2}}\left(\Delta Z_{11 k}-\theta_{a}\right)^{2}\right] d \varepsilon_{a}^{2}
\end{aligned}
$$

$$
\begin{aligned}
& p\left(\theta_{a} \mid \Delta Z_{11 k}\right) \\
& \propto \frac{\Gamma(n / 2)}{\left[\sum_{k=1}^{n}\left(\Delta Z_{11 k}-\theta_{a}\right)^{2} / 2\right]^{n / 2}} \cdot \int_{0}^{\infty} \frac{\left[\sum_{k=1}^{n}\left(\Delta Z_{11 k}-\theta_{a}\right)^{2} / 2\right]^{n / 2}}{\Gamma(n / 2)}\left(\varepsilon_{a}^{2}\right)^{-n / 2-1} \exp \left[\sum_{k=1}^{n}-\frac{1}{2 \varepsilon_{a}^{2}}\left(\Delta Z_{11 k}-\theta_{a}\right)^{2}\right] d \varepsilon_{a}^{2}
\end{aligned}
$$

The equation is finished as 


$$
=\frac{\Gamma(n / 2)}{\left[\sum_{k=1}^{n}\left(\Delta Z_{11 k}-\theta_{a}\right)^{2} / 2\right]^{n / 2}} .
$$

$\sum_{i=1}^{n}\left(\Delta Z_{11 k}-\theta_{a}\right)^{2}$ is transformed as $n\left(\Delta Z_{11 k}-\theta_{a}\right)^{2}+$ $\sum_{k=1}^{n}\left(\Delta Z_{11 k}-\Delta \bar{Z}_{11 k}\right)^{2}$ and then put it into (18):

$$
\begin{aligned}
p\left(\theta_{a} \mid \Delta Z_{11 k}\right) & \propto \frac{\Gamma(n / 2)}{\left[\sum_{k=1}^{n}\left(\Delta Z_{11 k}-\theta_{a}\right)^{2} / 2\right]^{n / 2}} \\
& \propto \frac{\Gamma(n / 2)}{\left[(1 / 2) \sum_{k=1}^{n}\left(\Delta Z_{11 k}-\Delta \bar{Z}_{11 k}\right)^{2}\right]^{n / 2} \cdot\left[1+n\left(\Delta \bar{Z}_{11 k}-\theta_{a}\right)^{2} / \sum_{k=1}^{n}\left(\Delta Z_{11 k}-\Delta \bar{Z}_{11 k}\right)^{2}\right]^{n / 2}} \\
& \propto\left[1+\frac{n\left(\Delta \bar{Z}_{11 k}-\theta_{a}\right)^{2}}{\sum_{k=1}^{n}\left(\Delta Z_{11 k}-\Delta \bar{Z}_{11 k}\right)^{2}}\right]^{-n / 2} \propto\left[1+\frac{\left(\theta_{a}-\Delta \bar{Z}_{11 k}\right)^{2}}{(n-1) S^{2} / n}\right]^{-n / 2},
\end{aligned}
$$

where $\Delta \bar{Z}_{11 k}=(1 / n) \sum_{k=1}^{n} \Delta Z_{11 k}, S^{2}=(1 /(n-1)) \sum_{k=1}^{n}\left(\Delta Z_{11 k}-\right.$ $\left.\Delta \bar{Z}_{11 k}\right)^{2}$. From (19), it can be seen that the marginal posterior distribution of $\theta_{1}$ follows the mean value of $\Delta \bar{Z}_{11 k}$, and the scale parameter fits the normal distribution of $S^{2} / n$.

Update the estimated parameter $\varepsilon_{a}^{2}$ in

$$
\begin{aligned}
p\left(\varepsilon_{a}^{2} \mid \Delta Z_{11 k}\right) & \propto \int_{0}^{\infty}\left(\varepsilon_{a}^{2}\right)^{-n / 2-1} \exp \left[-\frac{1}{2 \varepsilon_{a}^{2}} \sum_{k=1}^{n}\left(\Delta Z_{11 k}-\theta_{a}\right)^{2}\right] d \theta_{a} \\
& \propto\left(\varepsilon_{a}^{2}\right)^{-n / 2-1} \int_{0}^{\infty} \exp \left[-\frac{1}{2 \varepsilon_{a}^{2}}\left(n\left(\Delta Z_{11 k}-\theta_{a}\right)^{2}+\sum_{k=1}^{n}\left(\Delta Z_{11 k}-\Delta \bar{Z}_{11 k}\right)^{2}\right)\right] d \theta_{a} \\
& \propto\left(\varepsilon_{a}^{2}\right)^{-n / 2-1} \int_{0}^{\infty} \exp \left[-\frac{\left(\Delta Z_{11 k}-\theta_{a}\right)^{2}}{2 \varepsilon_{a}^{2} / n}-\frac{S^{2}}{2 \varepsilon_{a}^{2} /(n-1)}\right] d \theta_{a} \\
& \propto\left(\varepsilon_{a}^{2}\right)^{-n / 2-1} \cdot \exp \left[-\frac{(n-1) S^{2}}{2 \varepsilon_{a}^{2}}\right] \cdot \int_{-\infty}^{+\infty} \exp \left[-\frac{\left(\Delta \bar{Z}_{11 k}-\theta_{a}\right)^{2}}{2 \varepsilon_{a}^{2} / n}\right] d \theta_{a} \propto\left(\varepsilon_{a}^{2}\right)^{-n / 2-1} \cdot \exp \left[-\frac{(n-1) S^{2}}{2 \varepsilon_{a}^{2}}\right] .
\end{aligned}
$$

From (20), it can be seen that the posterior distribution of $\varepsilon_{a}^{2}$ is proportional to the probability density function of the inverse Gamma distribution. The shape parameter is $(n-1) / 2$ and the scale parameter is $(n-1) S^{2} / 2$. Based on (19) and (20), the mean value of the posterior distribution of $\theta_{1}$ is $\Delta \bar{Z}_{11 k} / \Delta t_{11 k}$, and the scale parameter is $s^{2} \Delta t_{11 k} / n$. The shape parameter of the posterior distribution of $\varepsilon_{1}{ }^{2}$ is $(n-1) / 2$, and the scale parameter is $(n-1) S^{2} / 2 \Delta t_{11 k}$. The estimated values of the parameters $\widehat{\theta}_{1}$ and $\widehat{\varepsilon}_{1}^{2}$ under the stress of $T_{1}$ are

$$
\begin{aligned}
& \widehat{\theta}_{1}=\frac{1}{m} \sum_{j=1}^{m} \theta_{1} ; \\
& \widehat{\varepsilon}_{1}^{2}=\frac{1}{m} \sum_{j=1}^{m} \varepsilon_{1}{ }^{2} .
\end{aligned}
$$


Substitute the estimated value of (21) into (23):

$$
\widehat{E}_{i j}=\frac{\left(\ln \widehat{\theta}_{i}-\ln \widehat{\theta}_{j}\right)-3\left[\ln \left(H_{R j}\right)-\ln \left(H_{R i}\right)\right]}{1 / K\left(273+T_{i}\right)-1 / K\left(273+T_{j}\right)} .
$$

The estimated value of $E$ can be calculated as

$$
\widehat{E}=\frac{1}{l(l-1)} \sum_{i=1}^{l} \sum_{j=1, i \neq j}^{l} \widehat{E}_{i j} .
$$

Based on (8), the estimated value of $A$ is

$$
\widehat{A}=\frac{1}{l} \sum_{i=1}^{l} \frac{\widehat{\theta}_{i}}{\left(H_{R i}\right)^{-3} \exp \left[\widehat{E} / K\left(273+T_{i}\right)\right]} .
$$

Since the diffusion coefficient $\varepsilon$ is independent of the acceleration stress, its estimated value is

$$
\widehat{\varepsilon}^{2}=\frac{1}{l} \sum_{i=1}^{l} \widehat{\varepsilon}_{i}^{2}
$$

After the values of the parameters in the degradation model have been obtained, the mean and variance of the degradation data under normal stress level can be obtained according to the accelerated degradation model. And the corresponding failure distribution can be obtained by substituting them into (6).

\subsection{Residual Life Prediction Model Based on Evidential Rea-} soning. As mentioned in Section 1, considering the advantages of evidential reasoning in theoretical research and practical application, the prediction model of residual life of complex systems based on evidential reasoning is established.

The core of the ER rule is a kind of distributed type with a belief structure. In this way, any input information, whether it is accurate data or inaccurate date with uncertainty, subjective or objective judgment, can be consistently modeled based on the power set. Moreover, the ER rule also handles with the probabilistic uncertainty by integrating the reliability of all indicators in (24). The details are as follows.

(1) Transformation of All Kinds of Input Indicators. On the one hand, different indicators can be described in different ways. On the other hand, there is always ignorance and vagueness in the prediction of residual life of complex systems. In addition, both qualitative and quantitative information are considered with uncertainties [25]. So, it is of much importance to transform various types of health indicators into belief distribution.

Define $N$ distinctive residual life grades as represented by

$$
H=\left\{H_{1}, H_{2}, \ldots, H_{i}, \ldots, H_{N}\right\}
$$

where $H_{i}$ is the $i$ th residual life grade. It is assumed that $H_{i+1}$ is inferior to $H_{i}$. In addition, $H$ could be determined by circumstances, including special conditions and personal experience. For example, three grades "Long" $\left(H_{1}\right)$, "Average" $\left(\mathrm{H}_{2}\right)$, and "Short" $\left(\mathrm{H}_{3}\right)$ can be chosen in the prediction of residual life of complex systems.

Thus, the distribution can be described as

$$
\begin{aligned}
S\left(x_{j}\right)=\left\{\left(H_{i}, \beta_{i, j}\right), i=1,2, \ldots, N\right\}, & \\
& j=1,2, \ldots, m,
\end{aligned}
$$

where $0 \leq \beta_{i, j}, \sum_{i=1}^{N} \beta_{i, j} \leq 1$, and $\beta_{i, j}$ denotes a degree of belief. The above distributed assessment reads that the indicator $x_{j}$ is assessed to the grade $H_{i}$ with the degree of belief $\beta_{i, j}$, which can be calculated as

$$
\begin{aligned}
\beta_{i, j}\left(x_{j}\right) & =\frac{U\left(H_{i+1}\right)-x_{i}}{U\left(H_{i+1}\right)-U\left(H_{i}\right)}, \\
U\left(H_{i}\right) \leq x_{i} \leq U\left(H_{i+1}\right) & \\
\beta_{i+1, j}\left(x_{j}\right) & =1-\beta_{i, j}\left(x_{j}\right), \quad U\left(H_{i}\right) \leq x_{j} \leq U\left(H_{i+1}\right) \\
\beta_{k, j}\left(x_{j}\right) & =0, \quad k=1,2, \ldots, N, k \neq i, i+1,
\end{aligned}
$$

where $k=1,2, \ldots, N-1, U\left(H_{i}\right)$ denotes a quantitative value that can be judged as a referential value $H_{i}$.

(2) Integration of Multi-Indicators. For complex systems, the residual life at time $t$ relies on all health indicators, which means the residual life can be obtained through multiple health indicators. The information in (28) can be integrated by the ER rule. The results of the residual life prediction $z(t)$ can be expressed as follows:

$$
L(z(t))=\left\{\left(H_{i}, \beta_{i}(t)\right), i=1,2, \ldots, N\right\}
$$

where the distribution $L(z(t))$ represents the assessment of the residual life of complex systems at time $t$, from which the detailed life grades and belief degrees can be seen clearly. $\beta_{i}(t), i=1,2, \ldots, N$ can be calculated as follows [15]:

$$
\begin{aligned}
& \beta_{i}(t)=\frac{\mu(t) \times\left[\prod_{l=1}^{n}\left(\bar{\omega}_{l}(t) \beta_{i, l}(t)+1-\bar{\omega}_{l}(t) \sum_{k=1}^{N} \beta_{k, l}(t)\right)\right]}{1-\mu(t) \times\left[\prod_{l=1}^{n}\left(1-\bar{\omega}_{l}(t)\right)\right]}-\frac{\mu(t) \times\left[\prod_{l=1}^{n}\left(1-\bar{\omega}_{l}(t) \sum_{k=1}^{N} \beta_{k, l}(t)\right)\right]}{1-\mu(t) \times\left[\prod_{l=1}^{n}\left(1-\bar{\omega}_{l}(t)\right)\right]} \\
& \mu(t)=\left[\sum_{i=1}^{N} \prod_{l=1}^{n}\left(\bar{\omega}_{l}(t) \beta_{i, l}(t)+1-\bar{\omega}_{l}(t) \sum_{k=1}^{N} \beta_{k, l}(t)\right)-(N-1) \prod_{l=1}^{n}\left(1-\bar{\omega}_{l}(t) \sum_{k=1}^{N} \beta_{k, l}(t)\right)\right]^{-1} \\
& \bar{\omega}_{l}(t)=\frac{\omega_{l}(t)}{1+\omega_{l}(t)-r_{l}},
\end{aligned}
$$


where $\omega_{l}(t)$ denotes the weight coefficient of the $l$ th indicator at time $t$ and $r_{l}$ denotes the reliability of the $l$ th indicator.

(3) Utility Evaluation. In order to visually represent the distributed assessment results, the expected utility is introduced. $u\left(H_{i}\right), i=1,2, \ldots, N$ is the utility of evaluation grade $H_{i}$, and the expected utility of the prediction result $L(z(t))$ is defined as follows:

$$
u(L(z(t)))=\sum_{i=1}^{N} \beta_{i}(t) u\left(H_{i}\right) .
$$

(4) Ways to Calculate the Adaptive Weighting Coefficient. The weighting coefficient represents the importance of the indicators for prediction results $[15,26]$. In this paper, the adaptive weight is calculated with CVBW method [27] shown as follows.

Suppose the observed data of indicator $x_{j}$ within $t$ is denoted by $z_{j}(k), k=1,2, \ldots, t$, whose mean value is $\bar{z}_{j}$. So, $\bar{z}_{j}$ can be calculated by

$$
\bar{z}_{j}=\frac{1}{t} \sum_{k=1}^{t} z_{j}(k) .
$$

$s_{z_{j}}$ denotes the mean square error of the observed data of indicator $x_{j}$ within time $t$, which can be calculated by

$$
s_{z_{j}}=\left[\frac{1}{t-1} \sum_{k=1}^{t}\left(z_{j}(k)-\bar{z}_{j}\right)^{2}\right]^{1 / 2} .
$$

Also, there is

$$
v_{z_{j}}=\frac{s_{z_{j}}}{\overline{z_{j}}} .
$$
by

The weighting coefficient of $x_{j}$ within $t$ can be calculated

$$
\omega_{j}(t)=\frac{v_{z_{j}}}{\sum_{j=1}^{n} v_{z_{j}}} .
$$

3.3. A New Algorithm for Residual Life Prediction of Complex Systems Based on Wiener Process and Evidential Reasoning. Based on the above analysis, a new algorithm for the residual life prediction of complex systems based on Wiener process and evidential reasoning is proposed in this section. The details are shown as follows.

Step 1. Construct the framework of the residual life prediction model of complex systems, and comb through the problems to be solved.

Step 2. Based on the working mechanism of complex systems, establish the degradation model of the systems, as is shown in (9).

Step 3. Estimate and update the parameters of the degradation model, using the maximum likelihood method and Bayesian method, respectively, as is shown in (21) and (27).

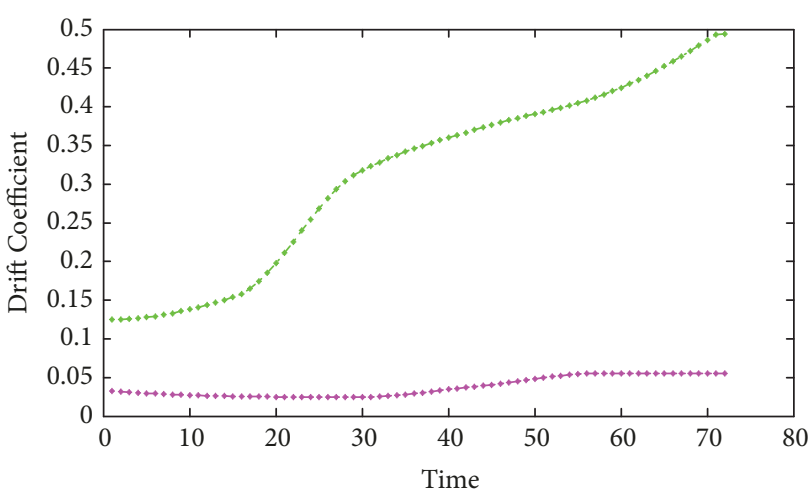

Figure 2: Degradation of the drift coefficient of gyroscope.

Step 4. Select the life indicators of complex systems, and obtain the residual life of complex systems based on Wiener process.

Step 5. Establish the residual life prediction model of complex systems based on evidential reasoning, as is shown in the Section 3.2.

Step 6. Calculate the adaptive weight, as is shown in (37). Based on the ER rule, make a precise prediction for the residual life prediction results of Wiener process.

\section{Case Study}

To prove the practicability of the proposed prediction models of complex systems, the residual life of gyroscope is studied in this paper. Considering the fact that the residual life of gyroscope is mainly determined by its drift coefficient which changes as time, the drift coefficient for zero and once term are chosen to measure the residual life of gyroscope.

4.1. Residual Life Prediction of Gyroscope Based on Wiener Process. Under the condition of constant temperature $20^{\circ} \mathrm{C}$ and humidity $70 \%$, the drift coefficient of the gyroscope is tested once a month, and the degradation values are obtained. To get more accurate results, the test is repeated. Figure 2 shows the results.

In Figure 2, Kox denotes the drift coefficient for zero item and $K s x$ denotes the drift coefficient for once item. Obviously, the two groups of degradation data are roughly linear, random processes, whose increments are not strict. Thus, the degradation process can be determined in Wiener process. In order to fit the data, the increment is introduced based on (4). According to the characteristics of Wiener process, $\Delta Z(t) \sim N\left(\theta \Delta t, \varepsilon^{2} \Delta t\right)$, it can be described by the probability distribution $\Delta Z(t)$. The fitting results are shown as Figures 3 and 4.

As can be seen from Figures 3 and 4, the drift coefficient degradation process of gyroscope is a Wiener process. Using the method described in the Section 3.1.2, four groups of degenerate data are updated to obtain the normal inverse Gamma posterior distribution parameters of $\theta$ and $\varepsilon^{2}$, which are shown in Table 1. 


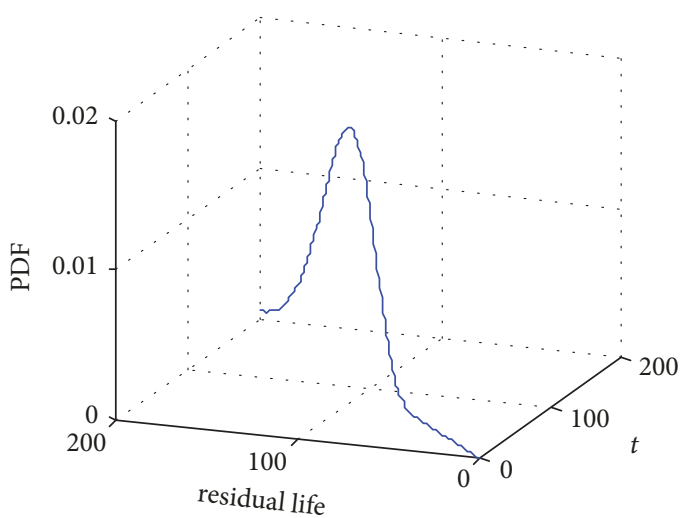

(a)

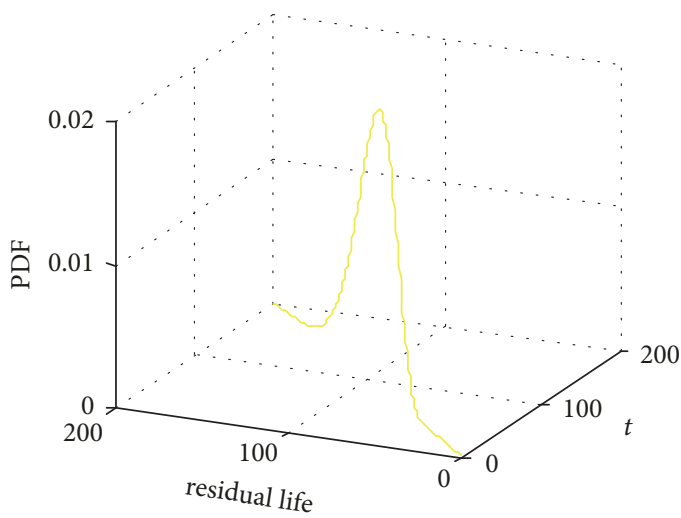

(c)

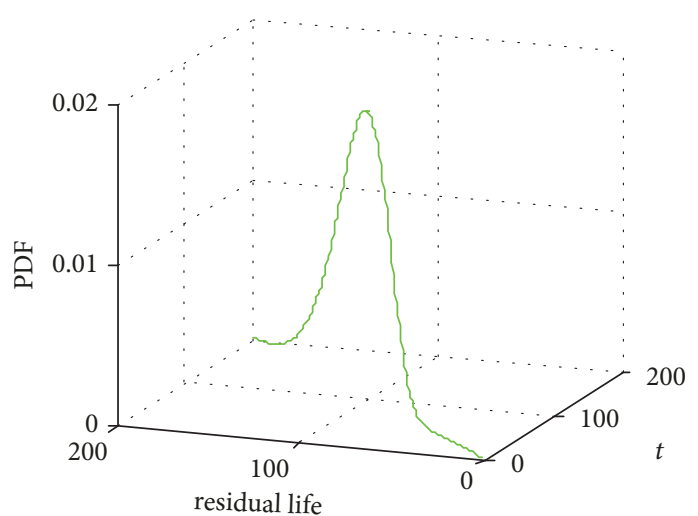

(b)

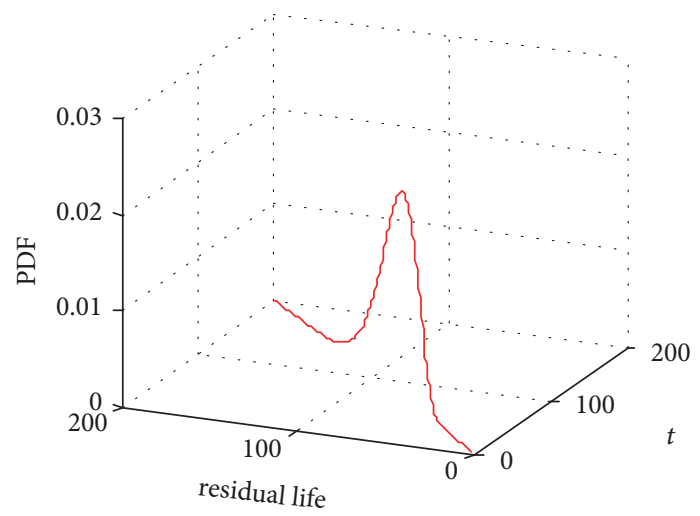

(d)

FIgURE 3: The degradation of Kox of gyroscope.

TABLE 1: The results of the estimated parameters.

\begin{tabular}{lcc}
\hline $\begin{array}{l}\text { Estimated } \\
\text { parameters }\end{array}$ & Drift coefficient $\hat{\theta}$ & $\begin{array}{c}\text { Diffusion coefficient } \\
\varepsilon^{2}\end{array}$ \\
\hline Kox & 0.0032 & 0.0072 \\
Ks $x$ & 0.0026 & 0.0018 \\
\hline
\end{tabular}

Remark 4. The parameters are set as shown in Table 2.

Finally, the prediction results of the residual life of gyroscope based on Kox and $K s x$ are obtained, which are shown in Tables 3 and 4, respectively.

According to Tables 3 and 4, the prediction results of the residual life of gyroscope based on Kox and $K s x$ are close to the real value, which has proved the accuracy of Wiener process in this paper. Therefore, the method proposed in the Section 3.1 can provide a reference for predicting the residual life of gyroscope in engineering practice.

Remark 5. Based on the actual degradation of gyroscope, the residual life of gyroscope is generally thought to be linear decreasing tendency.

\subsection{Residual Life Prediction of Gyroscope Based on Evidential} Reasoning. Based on evidential reasoning, the prediction results of the residual life of Wiener process are integrated. The details are as follows.
Firstly, calculate the weighting coefficient. The results are shown in Table 5.

Secondly, calculate the evidence reliability. Based on expert knowledge, the reliability of Kox and $K s x$ are 0.8262 and 1 , respectively. In addition, the assessment grades are set as "Very Long" $\left(H_{1}\right)$, "Long" $\left(H_{2}\right)$, "Average" $\left(H_{3}\right)$, "Short" $\left(H_{4}\right)$, and "Very Short" $\left(H_{5}\right)$, shown as follows.

$$
\begin{aligned}
H & =\left\{H_{1}, H_{2}, H_{3}, H_{4}, H_{5}\right\} \\
& =\{\text { Very Long, Long, Average, Short, Very Short }\} .
\end{aligned}
$$

In general, if the total life of gyroscope is 200 hours, then $H_{1}$ denotes the residual life for 200 hours, $H_{2}$ for $150, H_{3}$ for $100, H_{4}$ for 50 , and $H_{5}$ for 0 .

The standard values of each grade are 200, 150, 100, 50, and 0 , respectively, denoted by $A, B, C, D$, and $E$. Hence, the evidence is shown in Table 6.

Thirdly, integrate the evidence. Based on the method in the Section 3.2, the integration results are shown in Table 7.

It can be seen from Table 7 that when the initial value of the degradation of the gyroscope is 0.10 , the probability that the residual life of gyroscope is in the state $H_{1}$ is 0.0283 , and the probability of being in state $H_{2}$ is 0.9717 . So, the belief distribution can be described as follows:

$$
\begin{aligned}
& L(z(0.10))=\left\{\left(H_{1}, 0.0283\right),\left(H_{2}, 0.9717\right),\left(H_{3}, 0\right),\right. \\
& \left.\quad\left(H_{4}, 0\right),\left(H_{5}, 0\right)\right\} .
\end{aligned}
$$


TABLE 2: Parameters setting.

\begin{tabular}{|c|c|c|c|}
\hline Parameters & Initial value of degradation & Threshold value & Maximum life \\
\hline (a) & 0.10 & \multirow{4}{*}{0.5} & \multirow{4}{*}{200} \\
\hline (b) & 0.15 & & \\
\hline (c) & 0.20 & & \\
\hline (d) & 0.25 & & \\
\hline
\end{tabular}

TABle 3: Prediction values of residual life based on Kox compared with the real value under different initial values of degradation.

\begin{tabular}{lccc}
\hline Initial value of degradation & Real value & Predictive value & PDF \\
\hline 0.10 & 160 & 120 & 0.0166 \\
0.15 & 140 & 104 & 0.0179 \\
0.20 & 120 & 88 & 0.0195 \\
0.25 & 100 & 72 & 0.0216 \\
\hline
\end{tabular}

TABLE 4: Prediction values of residual life based on $K s x$ compared with the real value under different initial values of degradation.

\begin{tabular}{lccr}
\hline Initial value of degradation & Real value & Predictive value & PDF \\
\hline 0.10 & 160 & 153 & 0.0462 \\
0.15 & 140 & 134 & 0.0494 \\
0.20 & 120 & 115 & 0.0533 \\
0.25 & 100 & 95 & 0.0585 \\
\hline
\end{tabular}

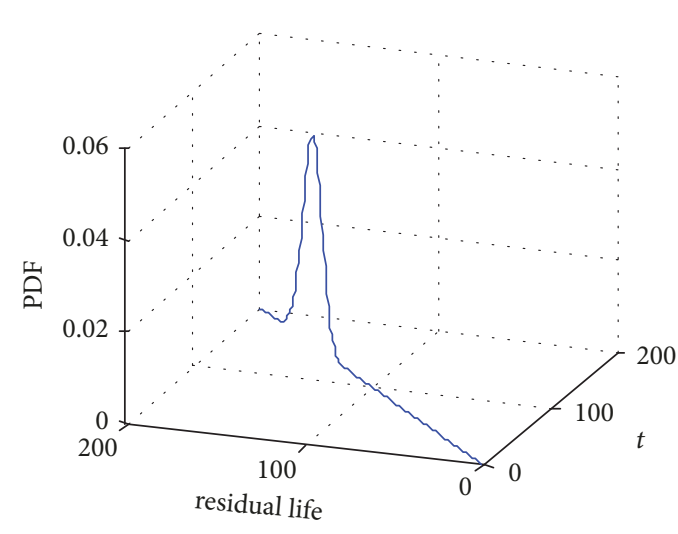

(a)

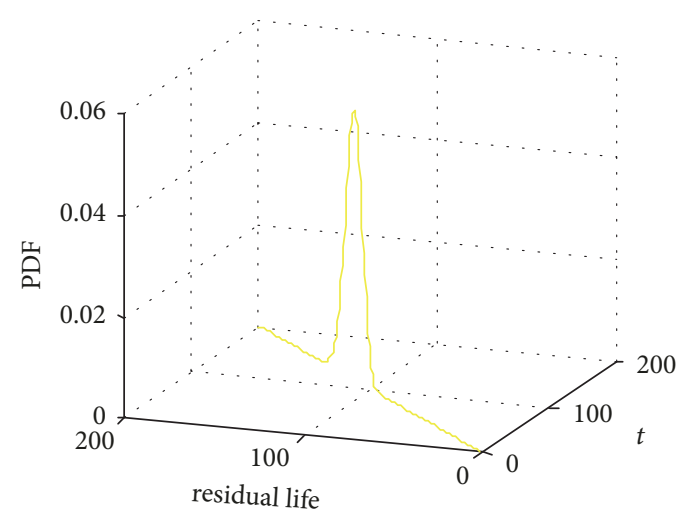

(c)

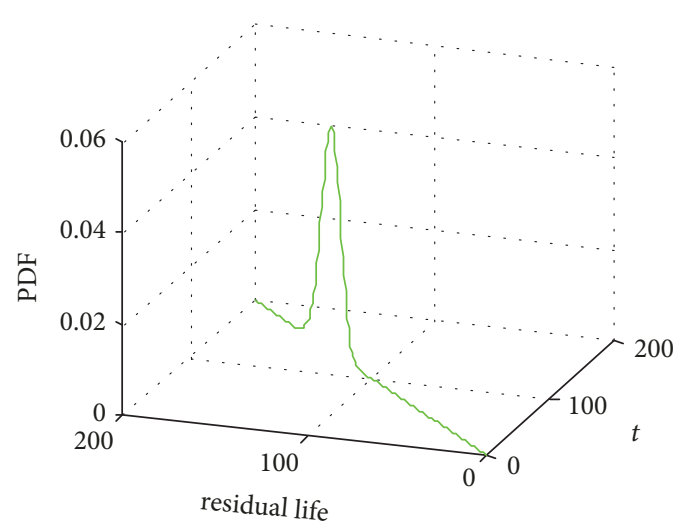

(b)

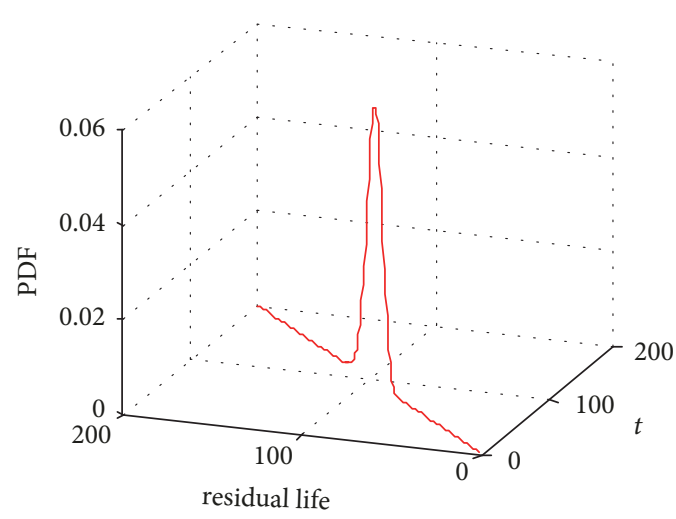

(d)

FIgURE 4: The degradation of $K s x$ of gyroscope. 
TABLE 5: The weighting coefficient of residual life of Kox and Ksx.

\begin{tabular}{lcccc}
\hline $\begin{array}{l}\text { Indicators } \\
\left(x_{j}\right)\end{array}$ & $\begin{array}{c}\text { Mean value } \\
\left(\overline{z_{j}}\right)\end{array}$ & $\begin{array}{c}\text { Mean square error } \\
\left(s_{z_{j}}\right)\end{array}$ & $\begin{array}{c}\text { Proportion } \\
\left(v_{z_{j}}\right)\end{array}$ & $\begin{array}{c}\text { Weighting coefficient } \\
\left(\omega_{j}(t)\right)\end{array}$ \\
\hline Kox & 96 & 20.6559 & 0.2152 & 0.5177 \\
$K s x$ & 124.25 & 24.9182 & 0.2005 & 0.4823 \\
\hline
\end{tabular}

TABLE 6: Two pieces of independent evidence.

\begin{tabular}{|c|c|c|c|c|c|c|}
\hline Initial value of degradation & $p_{\theta, i}$ & $A$ & $B$ & $C$ & $D$ & $E$ \\
\hline \multirow{2}{*}{0.10} & Kox & 0 & 0.4 & 0.6 & 0 & 0 \\
\hline & $K s x$ & 0.06 & 0.94 & 0 & 0 & 0 \\
\hline \multirow{2}{*}{0.15} & Kox & 0 & 0.08 & 0.92 & 0 & 0 \\
\hline & $K s x$ & 0 & 0.68 & 0.32 & 0 & 0 \\
\hline \multirow{2}{*}{0.20} & Kox & 0 & 0 & 0.76 & 0.24 & 0 \\
\hline & $K s x$ & 0 & 0.3 & 0.7 & 0 & 0 \\
\hline \multirow{2}{*}{0.25} & Kox & 0 & 0 & 0.44 & 0.56 & 0 \\
\hline & $K s x$ & 0 & 0 & 0.1 & 0.9 & 0 \\
\hline
\end{tabular}

TABLE 7: The integration results.

\begin{tabular}{|c|c|c|c|c|c|c|}
\hline $\begin{array}{l}\text { Initial value of } \\
\text { degradation }\end{array}$ & $\begin{array}{l}\text { Prediction } \\
\text { results }\end{array}$ & $H_{1}$ & $\mathrm{H}_{2}$ & $H_{3}$ & $H_{4}$ & $H_{5}$ \\
\hline \multirow[t]{2}{*}{0.10} & 120 & \multirow[t]{2}{*}{0.0229} & \multirow[t]{2}{*}{0.9771} & \multirow[t]{2}{*}{0} & \multirow[t]{2}{*}{0} & \multirow[t]{2}{*}{0} \\
\hline & 153 & & & & & \\
\hline \multirow{2}{*}{0.15} & 104 & \multirow{2}{*}{0} & \multirow{2}{*}{0.3654} & \multirow{2}{*}{0.6346} & \multirow{2}{*}{0} & \multirow{2}{*}{0} \\
\hline & 134 & & & & & \\
\hline \multirow[t]{2}{*}{0.20} & 88 & \multirow[t]{2}{*}{0} & \multirow[t]{2}{*}{0.0911} & \multirow[t]{2}{*}{0.9089} & \multirow[t]{2}{*}{0} & \multirow[t]{2}{*}{0} \\
\hline & 115 & & & & & \\
\hline \multirow[t]{2}{*}{0.25} & 72 & \multirow[t]{2}{*}{0} & \multirow[t]{2}{*}{0} & \multirow[t]{2}{*}{0.0862} & \multirow[t]{2}{*}{0.9138} & \multirow[t]{2}{*}{0} \\
\hline & 95 & & & & & \\
\hline
\end{tabular}

Therefore, we have a probability of 0.0283 that the residual life of gyroscope is very long, 0.9717 that the residual life is long, and 0 for other grades.

Similarly, the other results are shown as follows.

$$
\begin{aligned}
& L(z(0.15))=\left\{\left(H_{1}, 0\right),\left(H_{2}, 0.4130\right),\left(H_{3}, 0.5870\right),\right. \\
& \left.\quad\left(H_{4}, 0\right),\left(H_{5}, 0\right)\right\} \\
& L(z(0.20))=\left\{\left(H_{1}, 0\right),\left(H_{2}, 0.1161\right),\left(H_{3}, 0.8839\right),\right. \\
& \left.\quad\left(H_{4}, 0\right),\left(H_{5}, 0\right)\right\} \\
& L(z(0.25))=\left\{\left(H_{1}, 0\right),\left(H_{2}, 0\right),\left(H_{3}, 0.0878\right),\right. \\
& \left.\quad\left(H_{4}, 0.9122\right),\left(H_{5}, 0\right)\right\} .
\end{aligned}
$$

Therefore, the ER rule has well explained the prediction results of Wiener process, which is consistent with the actual observation results. In the engineering practice, when the drift coefficient of gyroscope is reduced to 0.25 , it can be considered that the residual life of gyroscope is very short, and a new gyroscope should be replaced for use.
Finally, all the above methods to predict the residual life of gyroscope are shown in Table 8 .

From Table 8, the common residual life prediction results of gyroscope can be obtained by Wiener process, while a specific prediction result can be obtained by the ER rule, which can integrate all of the former results.

To clearly demonstrate the superiority of the proposed methods, traditional fuzzy theory has been used to deal with the residual life prediction results of Wiener process [28-30]. Assuming that the set $U=\{A, B, C, D, E\}$, and that the fuzzy subsets are $K o x$ and $K s x$, respectively, based on Table 6, the membership of each subset is shown in Table 9.

Compared with Table 8, Table 9 shows that the final results obtained by fuzzy theory cannot be explained clearly, which are only some uncertain values. Therefore, the prediction results of the ER rule are proved to be more feasible and effective.

\section{Conclusion}

In this paper, a new model based on Wiener process and evidential reasoning is proposed to predict the residual life 
TABLE 8: The final results by ER.

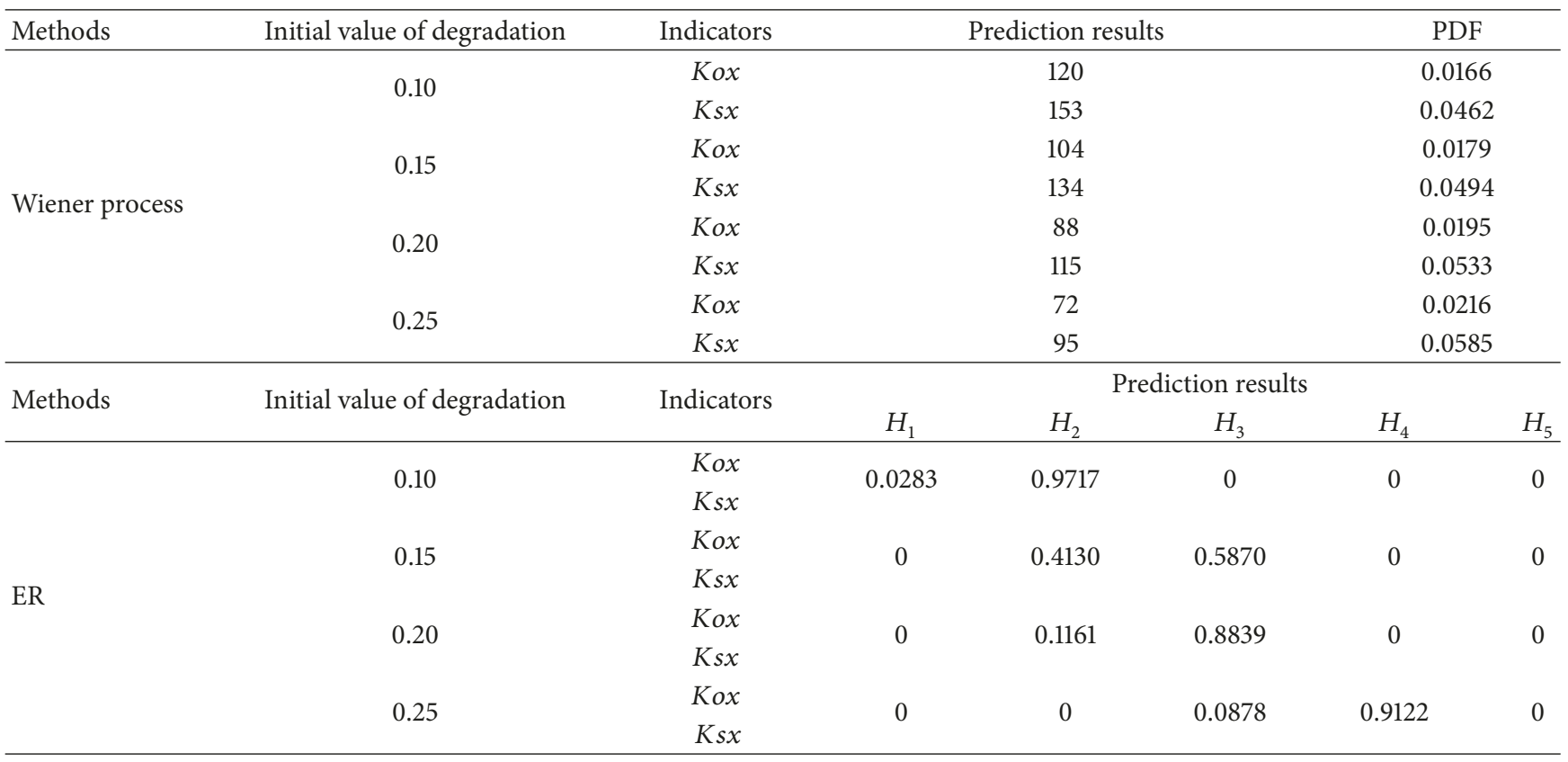

TABle 9: The membership of Kox and Ksx by fuzzy theory.

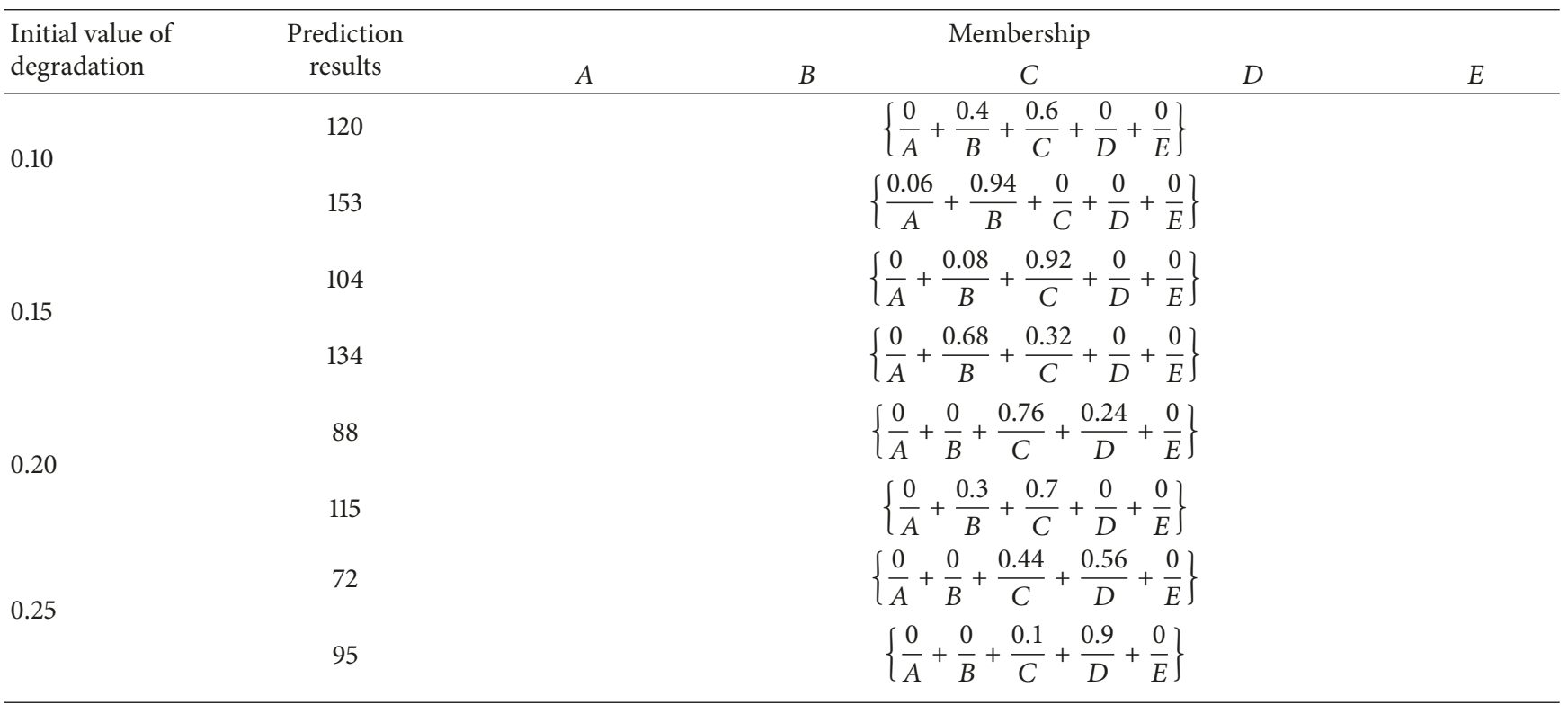

of complex systems. The relative importance of each health indicator is considered, which provides an effective method for predicting the residual life of complex systems. The model proposed in this paper is mainly composed of two parts that are prediction and integration. Through the case study of gyroscope and a comparison with fuzzy theory, it is proved more feasible and effective in engineering practice. As offline prediction proposed in this paper is unable to precisely and timely reflect the systems' life level, its effectiveness, and validity to deal with online problems and more complex problems should be further tested. At the same time, the reliability of all health indicators is crucial for the residual life prediction of the whole system and it is influenced by various factors, including the noise, sensor's characteristics, and malfunction. In addition, the correlation between each indicator needs to be considered. Hence, further study on how to obtain the reliability and correlation of indicators by integrating all factors based on online prediction needs to be done in the future.

\section{Conflicts of Interest}

The authors declare that they have no conflicts of interest. 


\section{References}

[1] Y. Q. Wang, H. Zhang, S. Wei, D. Zhou, and B. Huang, "Control performance assessment for ILC-controlled batch processes in two-dimensional system framework," IEEE Transactions on Systems, Man and Cybernetics: Systems, Article ID 2672563, 2017.

[2] Y. Wang, D. Zhao, Y. Li, and S. Ding, "Unbiased minimum variance fault and state estimation for linear discrete timevarying two-dimensional systems," Institute of Electrical and Electronics Engineers Transactions on Automatic Control, vol. 62, no. 10, pp. 5463-5469, 2017.

[3] C. S. Guo, Y. F. Zhang, N. Wan, R. Li, H. Zhu, and S. W. Feng, "The investigation of LED degradation model based on the chemical kinetics," Acta Physica Sinica, vol. 21, pp. 468-473, 2013.

[4] L. Liao and F. Köttig, "A hybrid framework combining datadriven and model-based methods for system remaining useful life prediction," Applied Soft Computing, vol. 44, pp. 191-199, 2016.

[5] Z.-Q. Wang, C.-H. Hu, W.-B. Wang, and G.-J. Dong, "Wiener process-based online prediction method of remaining useful life for draught fans in steel mills," Journal of University of Science and Technology Beijing, vol. 36, no. 10, pp. 1361-1368, 2014.

[6] Z.-Q. Wang, C.-H. Hu, W. Wang, and X.-S. Si, "An additive wiener process-based prognostic model for hybrid deteriorating systems," IEEE Transactions on Reliability, vol. 63, no. 1, pp. 208-222, 2014.

[7] Z. Q. Wang, C. H. Hu, W. Wang, Z. J. Zhou, and X. S. Si, "A case, study of remaining storage life prediction using stochastic filtering with the influence of condition monitoring," Reliability Engineering \& System Safety, vol. 132, no. 12, pp. 186-195, 2014.

[8] X. Wang, S. Lin, S. Wang, Z. He, and C. Zhang, "Remaining useful life prediction based on the Wiener process for an aviation axial piston pump," Chinese Journal of Aeronautics, vol. 29, no. 3, pp. 779-788, 2016.

[9] K. Hoshino, Y. Nishimura, Y. Yamashita, and D. Tsubakino, "Global asymptotic stabilization of nonlinear deterministic systems using Wiener processes," Institute of Electrical and Electronics Engineers Transactions on Automatic Control, vol. 61, no. 8, pp. 2318-2323, 2016.

[10] Q. Guan, Y. Tang, and A. Xu, "Objective Bayesian analysis accelerated degradation test based on Wiener process models," Applied Mathematical Modelling: Simulation and Computation for Engineering and Environmental Systems, vol. 40, no. 4, pp. 2743-2755, 2016.

[11] Z. Wang, J. Li, Y. Zhang, H. Fu, C. Liu, and S. Krishnaswamy, "A novel Wiener process model with measurement errors for degradation analysis," Eksploatacja i Niezawodność, vol. 18, no. 3, pp. 396-405, 2016.

[12] X. Wang, "Wiener processes with random effects for degradation data," Journal of Multivariate Analysis, vol. 101, no. 2, pp. 340-351, 2010.

[13] N. Cucu Laurenciu and S. D. Cotofana, "A nonlinear degradation path dependent end-of-life estimation framework from noisy observations," Microelectronics Reliability, vol. 53, no. 9-11, pp. 1213-1217, 2013.

[14] L. Zhu, H.-F. Zuo, and J. Cai, "Performance reliability prediction for civil aviation aircraft engine based on wiener process," Journal of Aerospace Power, vol. 28, no. 5, pp. 1006-1012, 2013.
[15] J.-B. Yang and D.-L. Xu, "Evidential reasoning rule for evidence combination," Artificial Intelligence, vol. 205, pp. 1-29, 2013.

[16] Z. J. Zhou, L. L. Chang, C. H. Hu, X. X. Han, and Z. G. Zhou, "A new BRB-ER-based model for assessing the lives of products using both failure data and expert knowledge," IEEE Transactions on Systems, Man, and Cybernetics, vol. 46, no. 11, pp. 1529-1543, 2016.

[17] Z. J. Zhou, C. H. Hu, G. Y. Hu, X. X. Han, B. C. Zhang, and Y. W. Chen, "Hidden behavior prediction of complex systems under testing influence based on semiquantitative information and belief rule base," IEEE Transactions on Fuzzy Systems, vol. 23, no. 6, pp. 2371-2386, 2015.

[18] L. Chang, Z. Zhou, Y. You, L. Yang, and Z. Zhou, "Belief rule based expert system for classification problems with new rule activation and weight calculation procedures," Information Sciences, vol. 336, no. 1, pp. 75-91, 2016.

[19] Z. J. Zhou, C. H. Hu, J. B. Yang, D. L. Xu, and D. H. Zhou, "Online updating belief-rule-base using the RIMER approach," IEEE Transactions on Systems, Man and Cybernetics, Part A: Systems and Humans, vol. 41, no. 6, pp. 1225-1243, 2011.

[20] Z. J. Zhou, C. H. Hu, and B. C. Zhang, "Hidden behavior prediction of complex systems based on hybrid information," IEEE Transactions on Cybernetics, vol. 43, no. 2, pp. 402-411, 2013.

[21] X. S. Si, C. H. Hu, and Z. J. Zhou, "Fault prediction model based on evidential reasoning approach," Science China Information Sciences, vol. 53, no. 10, pp. 2032-2046, 2010.

[22] V. Gitelman, E. Doveh, and S. Hakkert, "Designing a composite indicator for road safety," Safety Science, vol. 48, no. 9, pp. 1212$1224,2010$.

[23] J. Q. Liu, J. W. Xie, H. F. Zuo, and M. L. Zhang, "Remaining lifetime prediction for aeroengines based on Wiener process with random effects," Acta Aeronautica et Astronautica Sinica, vol. 02, pp. 564-574, 2015.

[24] X.-S. Si, W. Wang, C.-H. Hu, M.-Y. Chen, and D.-H. Zhou, "A Wiener-process-based degradation model with a recursive filter algorithm for remaining useful life estimation," Mechanical Systems and Signal Processing, vol. 35, no. 1-2, pp. 219-237, 2013.

[25] Z. G. Zhou, F. Liu, L. L. Li, Z. J. Zhou, J. B. Yang, and Z. L. Wang, "A cooperative belief rule based decision support system for lymph node metastasis diagnosis in gastric cancer," KnowledgeBased Systems, vol. 85, no. 9, pp. 62-70, 2015.

[26] D.-C. Ahn, J.-W. Lee, S.-J. Shin, and W.-J. Song, "A new robust variable weighting coefficients diffusion LMS algorithm," Signal Processing, vol. 131, pp. 300-306, 2017.

[27] F. J. Zhao, Z. J. Zhou, C. H. Hu, L. L. Chang, Z. G. Zhou, and G. L. $\mathrm{Li}$, "A new evidential reasoning-based method for online safety assessment of complex systems," IEEE Transactions on Systems, Man, and Cybernetics: Systems, pp. 1-13, 2016.

[28] J. Q. Zhang, "Sampling for building energy consumption with fuzzy theory," Energy and Buildings, vol. 156, pp. 78-84, 2017.

[29] B. Li, G. Yang, R. Wan, L. Zhang, Y. Zhang, and X. Dai, "Using fuzzy theory and variable weights for water quality evaluation in Poyang Lake, China," Chinese Geographical Science, vol. 27, no. 1, pp. 39-51, 2017.

[30] H.-C. Chang, S.-C. Lin, C.-C. Kuo, and C.-Y. Lin, "Fuzzy Theory-Based Partial Discharge Technique for Operating State Diagnosis of High-Voltage Motor," International Journal of Fuzzy Systems, vol. 18, no. 6, pp. 1092-1103, 2016. 


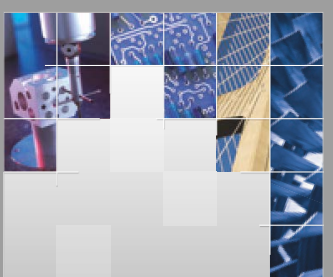

\section{Enfincering}
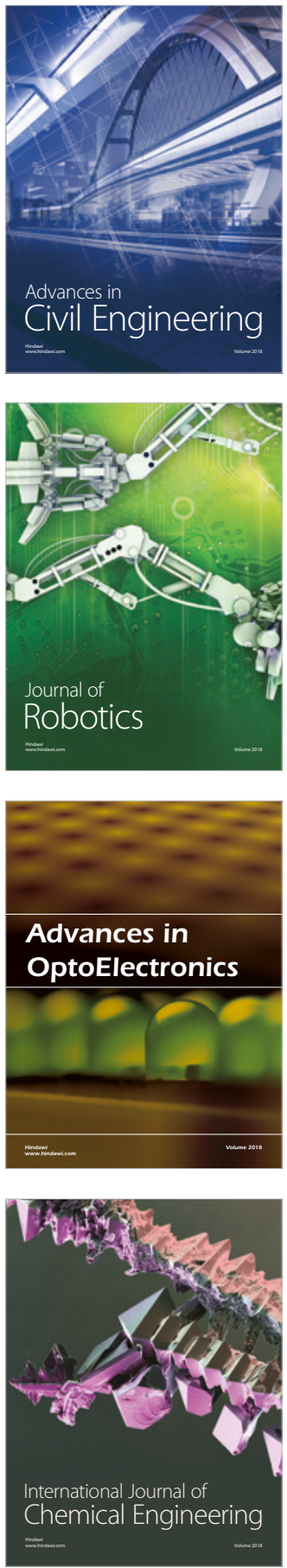

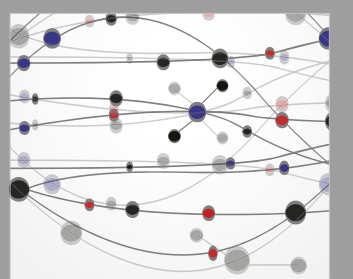

\section{Rotating \\ Machinery}

The Scientific World Journal

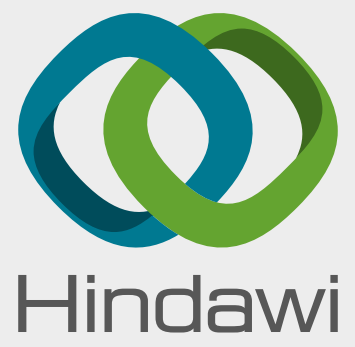

Submit your manuscripts at

www.hindawi.com
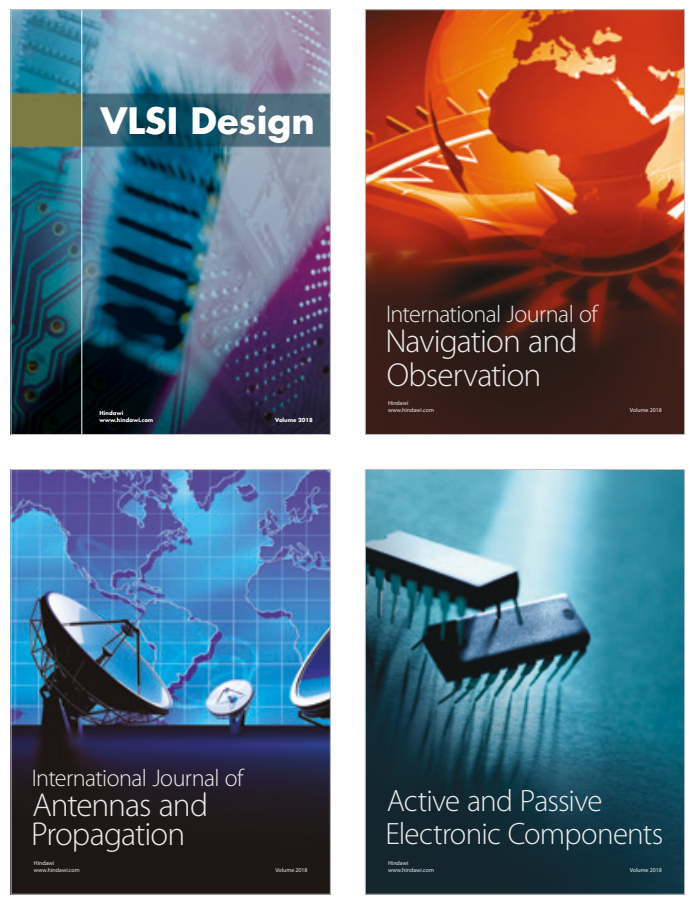
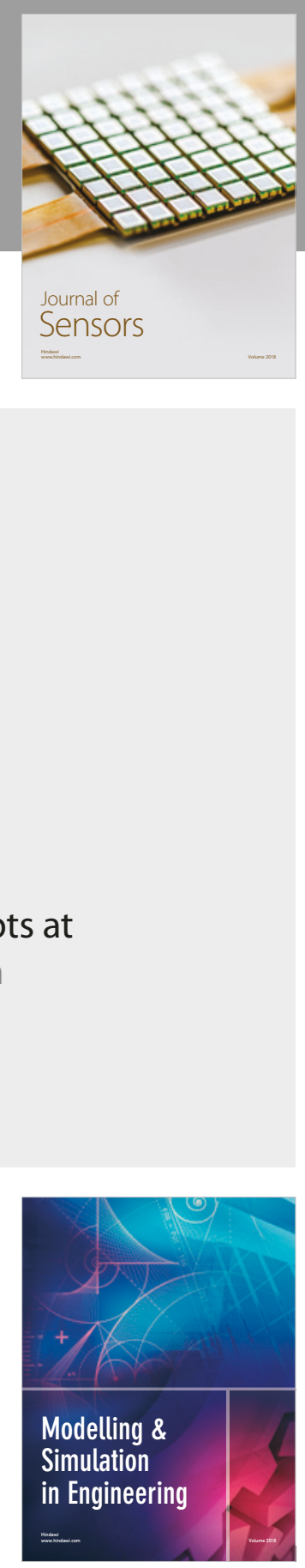

\section{Advances \\ Multimedia}
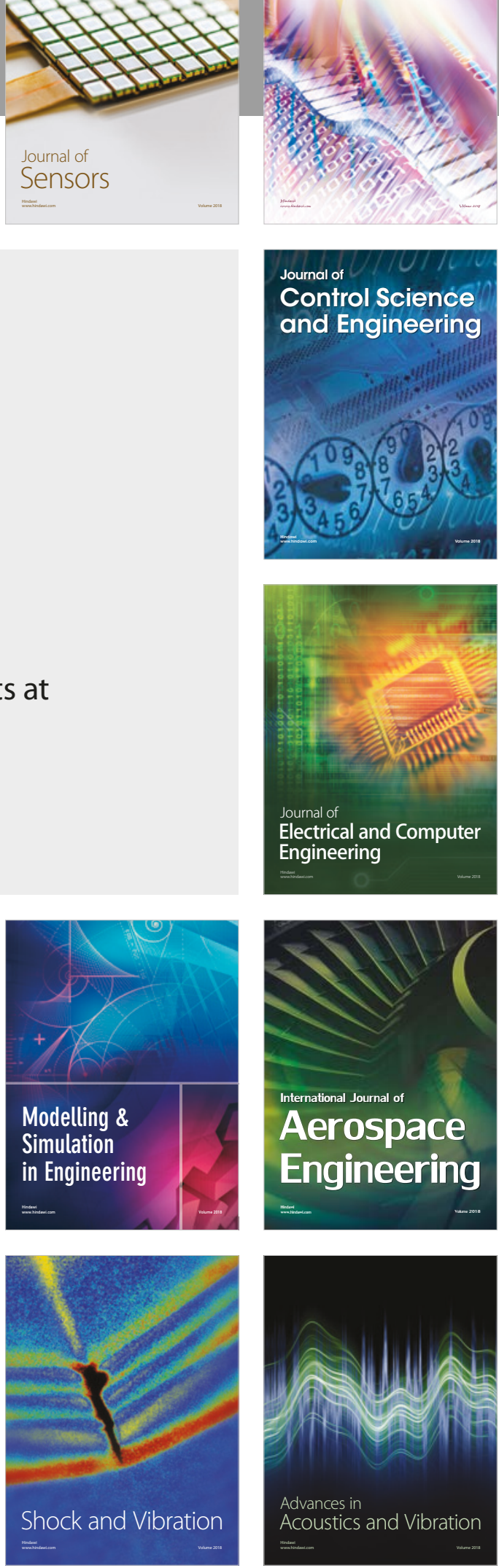